\title{
Using UHF-RFID Signals for Robot Localization Inside Pipelines
}

\author{
Amal Gunatilake ${ }^{\circledR}$, Mitchell Galea, Karthick Thiyagarajan ${ }^{\circledR}$, Sarath Kodagoda ${ }^{\circledR}$, \\ Lasitha Piyathilaka $^{\circledR}$, Poojaben Darji
}

\begin{abstract}
Underground water pipes are important to any country's infrastructure. Overtime, the metallic pipes are prone to corrosion, which can lead to water leakage and pipe bursts. In order to prolong the service life of those assets, water utilities in Australia apply protective pipe linings. Long-term monitoring and timely intervention are crucial for maintaining those lining assets. However, the water utilities do not possess the comprehensive technology to achieve it. The main reasons for lacking such technology are the unavailability of sensors and accurate robot localization technologies. Feature based localization methods such as SLAM has limited use as the application of liners alters the features and the environment. Encoder based localization is not accurate enough to observe the evolution of defects over a long period of time requiring unique defect correspondence. This motivates us to explore accurate contact-less and wireless based localization methods. We propose a cost-effective localization method using UHFRFID signals for robot localization inside pipelines based on Gaussian process combined particle filter. Experiments carried out in field extracted pipe samples from the Sydney water pipe network show that using the RSSI and Phase data together in the measurement model with particle filter algorithm improves the localization accuracy up to 15 centimeters precision.

Index Terms-infrastructure robotics, linings, localization, particle filter, pipes, robotics for smart cities, RFID, robotic inspections, UHF-RFID.
\end{abstract}

\section{INTRODUCTION}

Australia has over $250,000 \mathrm{~km}$ of water and wastewater pipelines. Corrosion is an important cause of pipe failures [1]. Depending on the size of the pipe, average corroded pipe repair costs range from $\mathrm{A} \$ 400$ to $\mathrm{A} \$ 4000$ per metre. There are several inspection methodologies based on pulsed Eddy current sensors [2], laser profilers and stereo vision [3], electrical resistivity sensors [4], fibre optic sensors [5], drilling mechanisms [6], ultrasound sensing [7], infrared radiometers [8] to name a few are currently used to identify hot spot corrosion zones in the water utility pipelines. Based on the level of pipe degradation, the water utilities either choose to replace that respective section of the pipe or apply protective pipe linings to extend the service life of the pipe section [9].

The application of linings in the water utility pipe infrastructures can deteriorate over time. For example, in drinking

This work was supported by the University of Technology Sydney (UTS) through the Faculty of Engineering and Information Technology (FEIT) Tech Lab Blue Sky Research Grant.

All the authors are with the iPipes Lab, UTS Robotics Institute, Faculty of Engineering and Information Technology, University of Technology Sydney, Sydney, New South Wales, 2007, Australia (e-mail: Amal.D.Gunatilake@student.uts.edu.au; Karthick.Thiyagarajan@uts.edu.au; $\quad$ Sarath.Kodagoda@uts.edu.au; lasithaya@gmail.com; Poojaben.Darji@student.uts.edu.au).

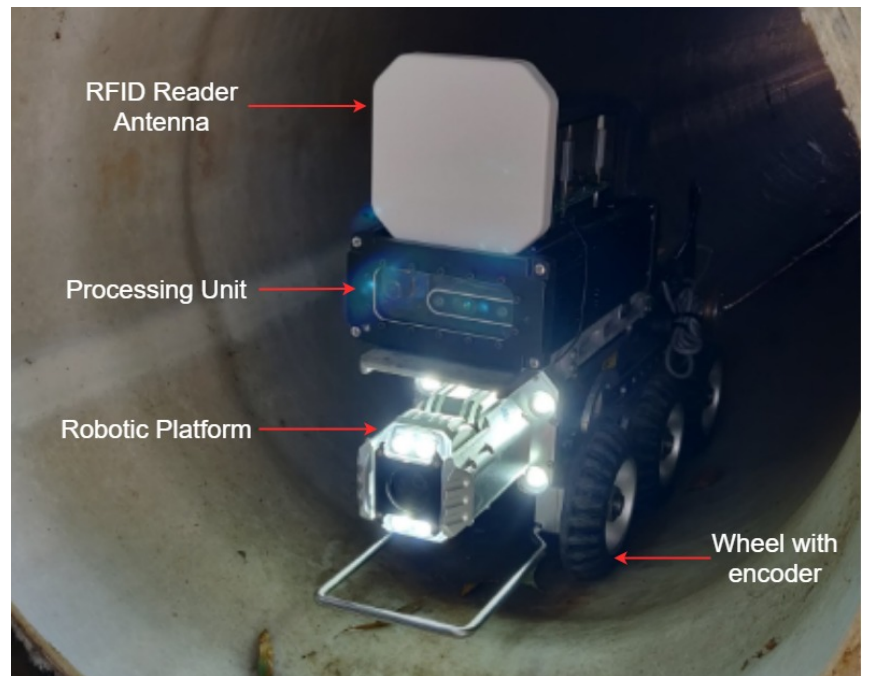

Fig. 1: Robot with RFID unit mounted on top.

water pipe infrastructure, liner imperfections are the most important defect to be monitored for post application quality assurance and long-term liner performance evaluation. Currently, we have developed a laser profiling robotic system for liner imperfection detection and quantification [10]. For effective long-term monitoring robust localization method is needed to compare the laser prdofiles taken at different time period to monitor liner imperfection defect evolution. However, due to localization errors of the robotic platforms, aligning these data after multiple deployments to analyze the defect evolution and subsequent intervening has become challenging. The most commonly used localization methods for in-pipe robotic platforms are wheel encoders or tether line encoders. The commonly used Simultaneous Localization and Mapping (SLAM) like localization techniques become obsolete due to the changing environmental conditions such as application of liners creating a completely different environment (features) and long term corrosion patches. When it comes to floating robots or drones in operational pipes, contact based localization methods become obsolete and therefore, a different and more accurate contactless localization method in pipelines is needed.

Currently, many different wireless technologies and algorithms being researched for localization in outdoor and indoor environments [11], [12]. However, there aren't many localization methods for underground pipelines. We have selected the radio-frequency identification (RFID) localization technology because it is more cost-effective and it is being 
used in liner embedded sensing technology to measure environmental conditions such as moisture and temperature in water pipelines [13]-[15], it can also be used as an effective contactless and wireless localization method. Further, it is not dependent on the environmental changes of the pipe over time. However, unlike outdoor RFID localization [16] which gives a straightforward signal curve that only contains one peak point [17] which are most likely location of the robot; inside pipelines the signal strength curve will have multiple peak points making the robot location uncertain. Further commercial off-the-shelf (COTS) RFID readers provides vague and uncertain measurements which is only capable of localizing the RFID tag with a square meter range accuracy [18]. Therefore the RFID localization task inside pipelines becomes unique.

In this paper, we proposed an improved localization method for underground water pipelines using COTS RFID components with particle filter algorithm [19], [20] that employs both Received Signal Strength Indication (RSSI) and Phase data in the measurement model to achieve higher accuracy. We have conducted experiments with field extracted water pipelines from the Sydney water network. We have done experiments to identify the best locations to deploy the RFID tags inside the pipe and the results show that our proposed system has achieved a precision of 15 centimeters.

The rest of this article is structured as follows: Section II describes the algorithms, hardware and software architectures. The experimental results are presented in Section III with discussions and finally, Section IV concludes the article by summarizing the key outcomes and briefs the intended future work.

\section{Methodology}

The initial experiments have shown in the open environment RFID localization is somewhat straight forward since the signal strength curve only contains one single peak point which is relating to the most likely location of the robot [17]. However inside a pipe environment the signal strength curve have multiple peak points making the robot location uncertain which makes the localization task unique and difficult. Results in Fig. 6 Tag A inside the pipe data and outside the pipe data comparison gives you an indication as to how it behaves differently in the two different environmental conditions.

We have introduced the particle filter for passive UHF RFID localization and we have improved the performance and accuracy of the localization by applying the Gaussian process data modeling into the RFID RSSI data. We have further improved the accuracy by embedding the Phase data into the measurement model.

First we collect RFID RSSI data and Phase data from the robot. The collected RSSI data shows a unique signal pattern which can be modeled using a Gaussian process. Therefore to filter the outliers, normalize the data and create a measurement model we use the Gaussian data modeling function.
Gausian Process regression modeling approach [21], [22] is used in this work to normalize the noisy data coming from the robotic measurements. The model obtains the distance travelled by the robot $D$ and predicts the RSSI values $(R)$. This can be learned as a function $f$ in the form of

$$
R=f(D)+\xi
$$

where $\xi$ is the uncertainty. Let $[X, Y]$ be the robotic measurement inputs where $X=\left[x_{1}, x_{2}, x_{3}, \ldots . ., x_{m}\right]^{T}, x_{i}=$ $\left[(D)_{i}\right]^{T}$, and $i(1 \leq i \leq m)$ is an integer and $m$ is the number of data pairs. $Y=\left[y_{1}, y_{2}, y_{3}, \ldots \ldots, y_{m}\right]^{T}$ is a vector having corresponding targets where $y_{i}=R_{i} \cdot\left[X^{*}, Y^{*}\right]$ is the testing data, where $X^{*}=\left[x_{1}^{*}, x_{2}^{*}, x_{3}^{*}, \ldots \ldots, x_{n}^{*}\right]^{T}$ is a matrix having robotic data inputs and $Y=\left[y_{1}^{*}, y_{2}^{*}, y_{3}^{*}, \ldots \ldots, y_{n}^{*}\right]^{T}$ is a vector having predicted outputs corresponding to $X^{*}$. Once $f$ has been learned using $[X, Y], f$ can be used to predict $Y^{*}$ for a given $X^{*}$ by using $D^{*}=f\left(\rho_{a}^{*}\right)+\xi^{*}$. A $K(X, X)$ kernel having $k_{i, j}=k\left(x_{i}, x_{j}\right)$ elements was selected to address the non-linear regression problem through GP modeling. In this work, we use the squared exponential kernel, which is defined as in (2).

$$
k\left(x_{i}, x_{j}\right)=\alpha^{2} \exp \left\{-\frac{1}{2 \beta^{2}}\left\|x_{i}-x_{j}\right\|^{2}\right\}
$$

where the $\alpha$ and $\beta$ are the hyper-parameters for the GP model. The GP model was trained by minimizing the negative $\log$ marginal likelihood in (3) with respect to $\theta=$ $\left\{\alpha, \beta, \sigma_{n}\right\}$. The covariance function $\sum$ is defined as:

$$
-\log p(Y \mid X, \theta)=\frac{1}{2} Y^{T}\left(\sum\right)^{-1} Y+\frac{1}{2}\left|\sum\right|+\frac{m}{2} \log (2 \pi)
$$

$$
\sum=K(X, X)+\sigma_{n}^{2} I
$$

The predicted RSSI values $\left(Y^{*}\right)$ for the distance $\left(X^{*}\right)$ will be given by the mean of the posterior distribution $(\mu)^{*}$ in (5) and the associated uncertainty is given by the covariance $\left(\sum\right)^{*}$ in (6).

$$
\begin{array}{r}
(\mu)^{*}=K\left(X^{*}, X\right)\left\{K(X, X)+\sigma_{n}^{2} I\right\}^{-1} y \\
\left(\sum\right)^{*}=K\left(X^{*}, X^{*}\right)+\sigma_{n}^{2} I-\left\{K\left(X^{*}, X\right)\right. \\
\left.K(X, X)+\sigma_{n}^{2} I\right\}^{-1} K\left(X, X^{*}\right)
\end{array}
$$

From the collected data, unlike the RSSI data that shows a unique signal pattern which can be mapped to a Gaussian process function, the Phase data is somewhat noisy (Fig. 7), therefore we are generating different weight sets to give high priority to the RSSI data and low priority to the Phase data and finally combine to create an improved measurement model. Then we map these data models with the odometry data from the robot to output the final measurement model.

Let, $R$ be the RSSI matrix model, $P$ be the Phase matrix model, $W_{1}$ be the wights generated for the RSSI matrix 


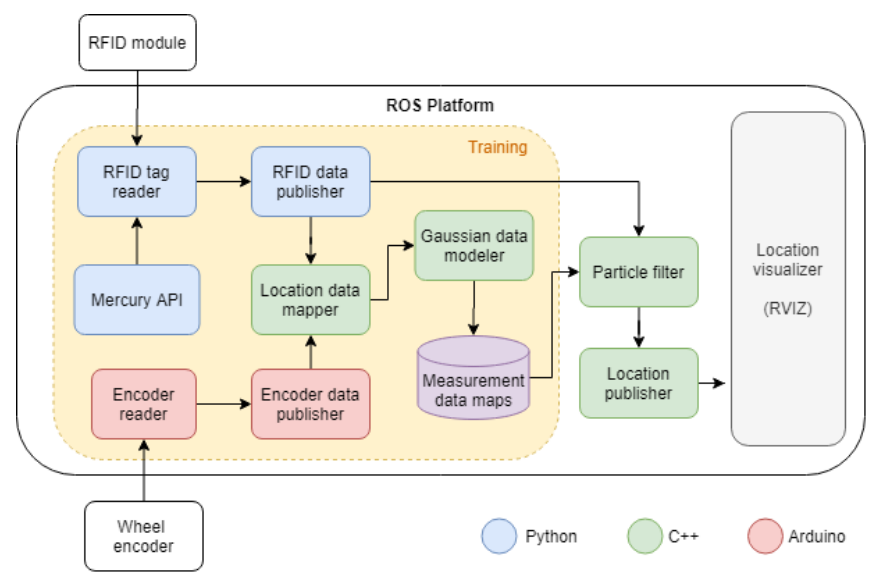

Fig. 2: Software architecture.

model and $W_{2}$ be the weights generated for Phase matrix model, that maps to $O$ odometry matrix data model received from the robot will denote the $M$ measurement model.

$$
M=\left[\begin{array}{c}
G(R) \\
P
\end{array}\right] \times\left[\begin{array}{ll}
W_{1} & W_{2}
\end{array}\right] \mapsto[O]
$$

Once we have the final measurement model from the initial robot deployment, Thereafter we can use this measurement model against the particle filter algorithm [19], [20] to predict the robot location. The highest prediction value will be the most likely location of the robot.

\section{A. Prototype development}

1) Sensor suite: The sensor suite was built with commercial off the shelf (COTS) RFID components. Thingmagic M6e Micro-LTE UHF 2 port RFID reader module with the embedded developer kit was used to implement the proposed system as it was easy to customize and it was actively supported by the open source Python MercuryAPI software community. A single $915 \mathrm{MHz}$ General Purpose Panel RF Antenna in $902 \mathrm{MHz}$ to $928 \mathrm{MHz}$ range with $5.5 \mathrm{dBi}$ gain was used to support the module. Three different most commonly available chip based RFID tag types (Fig. 5) were selected and used to conduct experiments to evaluate and identify the best suitable tag type for the proposed system to achieve best results. Jetson Nano Developer kit board with Quadcore ARM $1.43 \mathrm{GHz}$ CPU, 4 GB 64-bit LPDDR4 RAM was used as the central processing unit to run the implemented system. The whole hardware system was assembled inside an enclosure and mounted on top of a robotic platform for deployment Fig. 1. A rotary encoder with 1024 pulses per revolution was fixed to the robot wheels to collect the odometry data from the robot for validations.

2) Software architecture: The software architecture (Fig. 2) has been implemented on top of Robotic Operating System (ROS) framework. Since the RFID hardware module is supported with a Python Mercury API library, the RFID reader module has been implemented with Python. To gain more flexibility and structure to the implementation the core integration and algorithms were implemented as $\mathrm{C}++$ components. The robot wheel encoder electronics that receives the initial odometry data to create measurement maps for the particle filter has been implemented using Arduino components. Finally the estimated particle filter localization prediction which is based on the previously trained measurement maps are displayed on RVIZ like visualization systems. At the initial deployment, robot collects the RFID and odometry data to publish as ROS topics which will be used to generate the measurements models. In the next deployments, when the system doesn't have the wheel odometry data, particle filter can localize the robot using the RFID data and the measurement model.

\section{EXPERIMENTS \& RESULTS}

\section{A. Electromagnetic Field Simulation}

Fig. 4 shows the simulation results carried out by using the CST studio simulation software. The first simulation was carried out in the open environment by designing a UHF RFID antenna with hardware configurations that matched those of the actual antenna used in practical experiments. The signal strength of the antenna distributed evenly across the space as an expanding radial field that decays along the distance, as shown in the results (Fig. 4a). The same antenna was then moved inside a metallic pipe setting for the next simulation. The signal is reflected from the pipe surface and travels further, as seen in the results (Fig. 4b), giving the signal strength a ripple effect. This implies that signals move farther and behave differently within a pipe environment, making the RFID localisation task unique and challenging. Following the observation of these patterns, functional experiments were conducted to explain the findings.

\section{B. RFID tags \& location selection}

Chip based RFID tags were chosen for the experiments because they can be uniquely identified using the Electronic Product Code (EPC) value and they can be further used to store extra information. Mainly three different chip based UHF RFID tags (Fig. 5) were used to find the best suitable tag for improving the RFID localization results. The three different tag types are: oil impregnated paper based substrate with non coiled antenna (Fig. 5a), oil impregnated paper based coiled antenna (Fig. 5b), strong metalic based substrate with non coiled antenna (Fig. 5c). Fig. 6 shows the results comparison for different tags (Fig. 5) tested to identify the best suitable tag for localization. These tags were placed in the middle of the scanned length and the robot traveled from one end to the other. As seen in the results, it is clear that inside the pipe the signals travels far and has a reflected ripple effect. Inside the pipe, the data has more density than in the open space scan. Further, when the antenna passes the tags, the signal strength and the data density diminishes. It is observed that Tag A will perform better as it has low noise in the signal and a clear reflection pattern that will help better in the localization algorithms. Fig. 7 shows the RSSI and Phase data comparisons from a single tag. The RSSI data seems to have more unique signal pattern than 


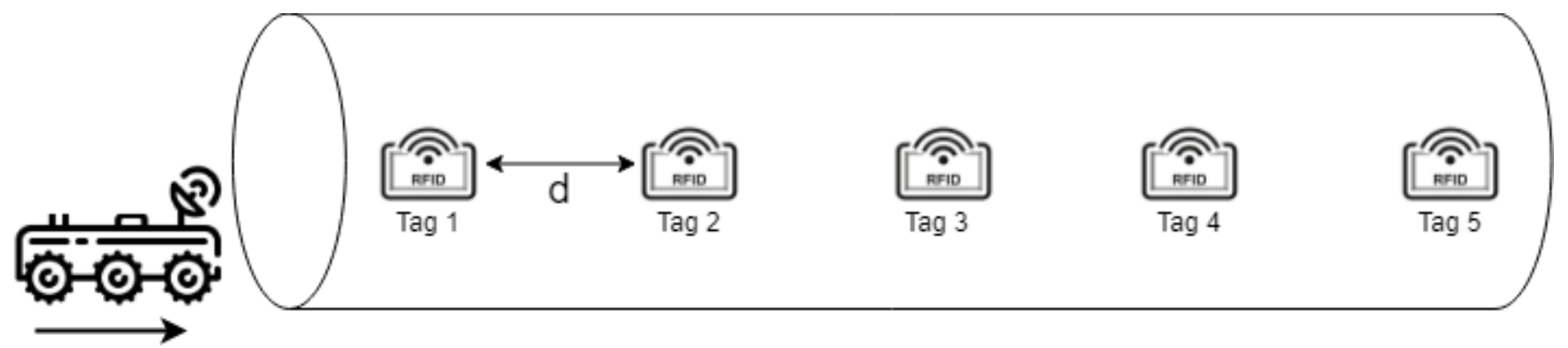

(a) RFID experiment setup inside pipeline.

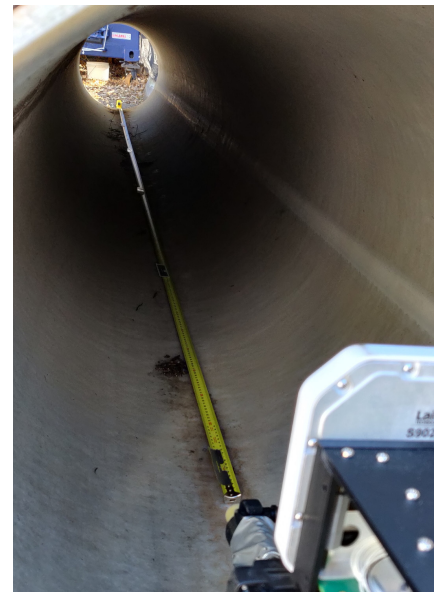

(b) RFID tags placed on the bottom of the pipe.

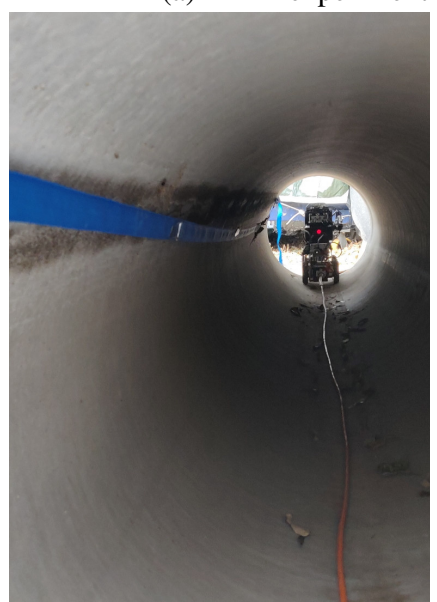

(c) RFID tags placed at the side of the pipe.
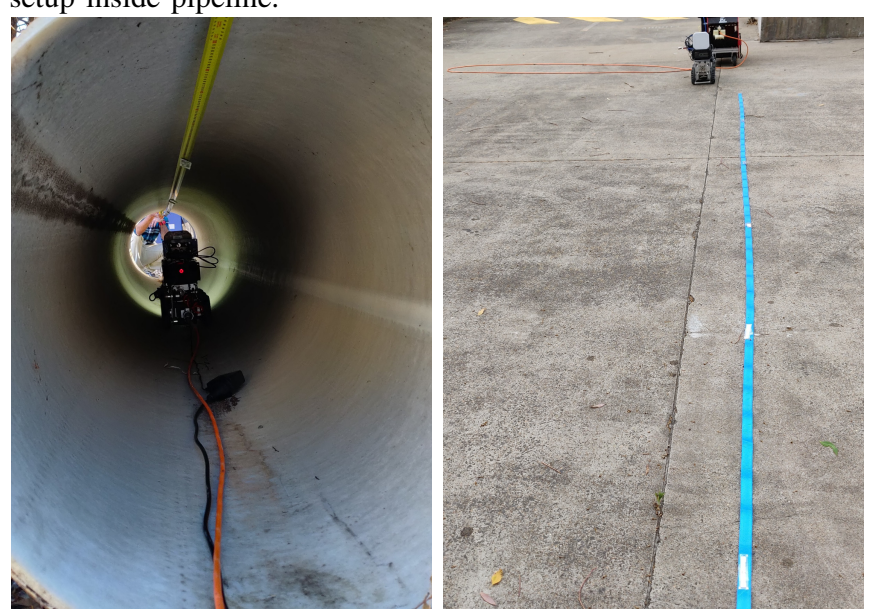

(e) RFID tags placed at an open area.

Fig. 3: RFID test setups.


(b) RFID antenna far-field sim(a) RFID reader antenna far-field ulation inside the pipeline envisimulation in open environment. ronment.

Fig. 4: CST studio RFID simulation results.

the phase data. Next, the same experiment was conducted in two different environments. Inside pipe environments and outside open environment to compare the behavior of the sensors. The tags were arranged inside a 6 meters long 600 millimeters in diameter field extracted water pipe sample as shown in Fig. 3 and data has been collected using the robot. This experiment was repeated by changing the RFID tag location inside the pipe surface as top, side and bottom (Fig. $3 d, 3 c, 3 b)$. Further the tests are repeated by changing the distance $(d)$ between each RFID tags. Fig. 8 shows the data collected by placing RFID tags in different locations in the pipe. The three scans were done by placing the Tag type A in the middle of the pipe. First scan shows when it placed in the bottom section of the pipe, next at the side of the pipe

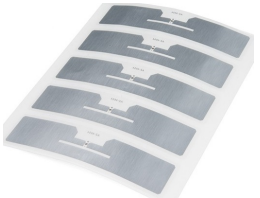

(a) Tag A.

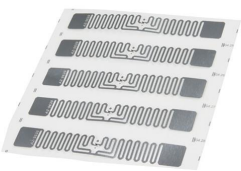

(b) Tag B.

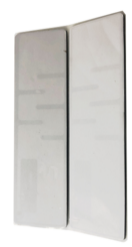

(c) Tag C.
Fig. 5: RFID tags.

and the last at the top crown of the pipe. The observations show that side is the best location to place the RFID tags.

\section{Data modeling}

The collected data was then mapped with the robot's odometry data to model the Gaussian process, which filters outliers and provides a stable data inputs for training the particle filter algorithm. Fig. 9 depicts the scan results of the 1 metre apart tag distribution inside the water pipeline sample as depicted in Fig. 3a, 3c. The red fitted curve represents the Gaussian process model, which normalises the raw data before it is used as the measurement model for the particle filter.

\section{System performance}

Using these data models, the particle filter based localization algorithm was tested in a ROS simulated environment 

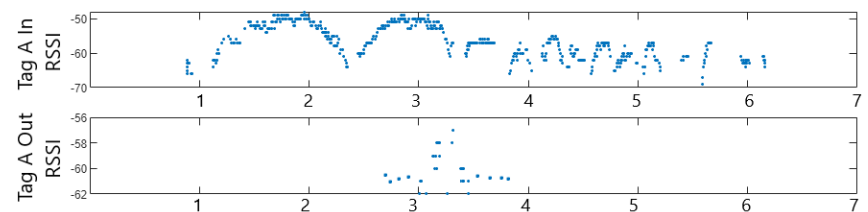

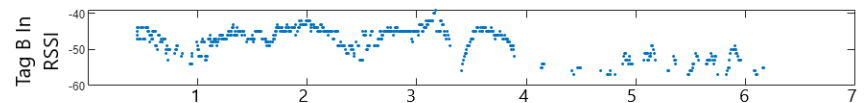
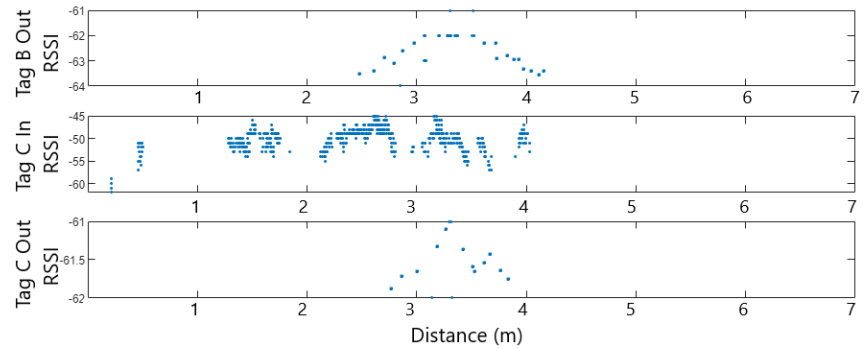

Fig. 6: Different RFID tags comparison.
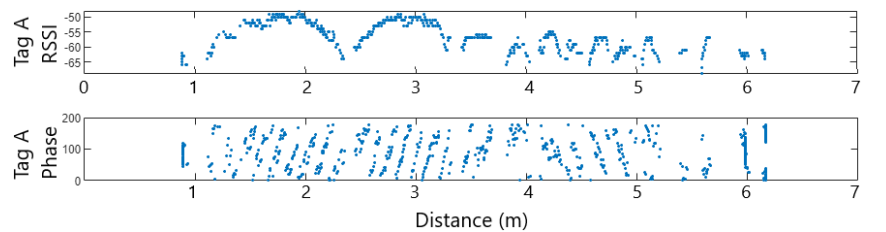

Fig. 7: RFID tag RSSI and Phase data.
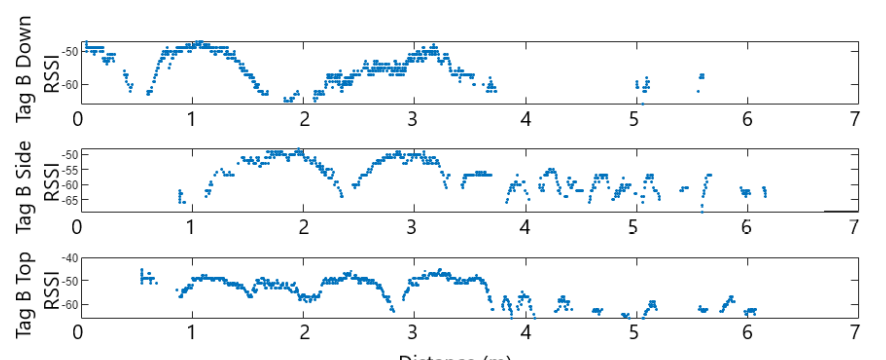

Distance $(\mathrm{m})$

Fig. 8: RFID tag location comparison.
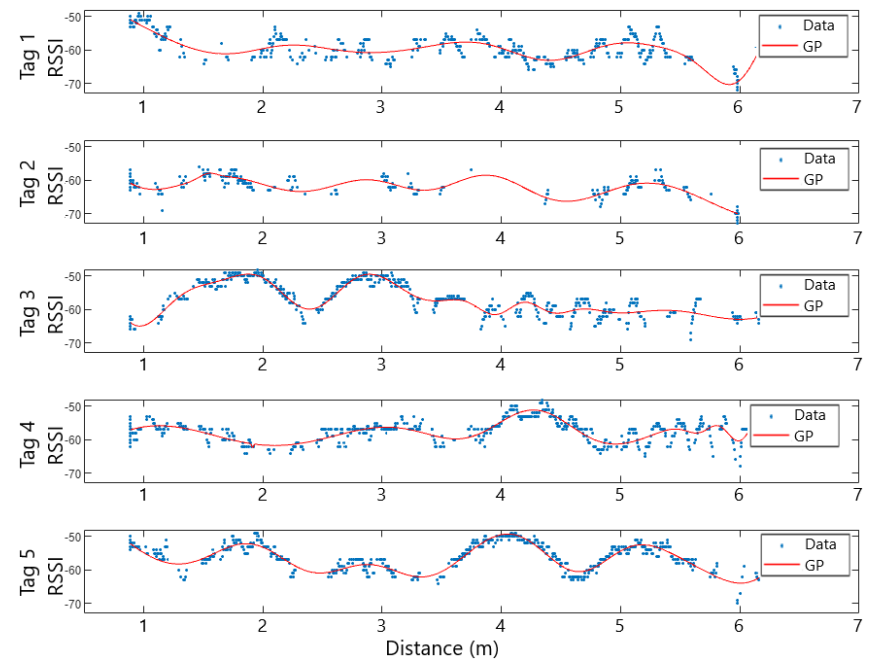

Fig. 9: RFID GP data modeling.

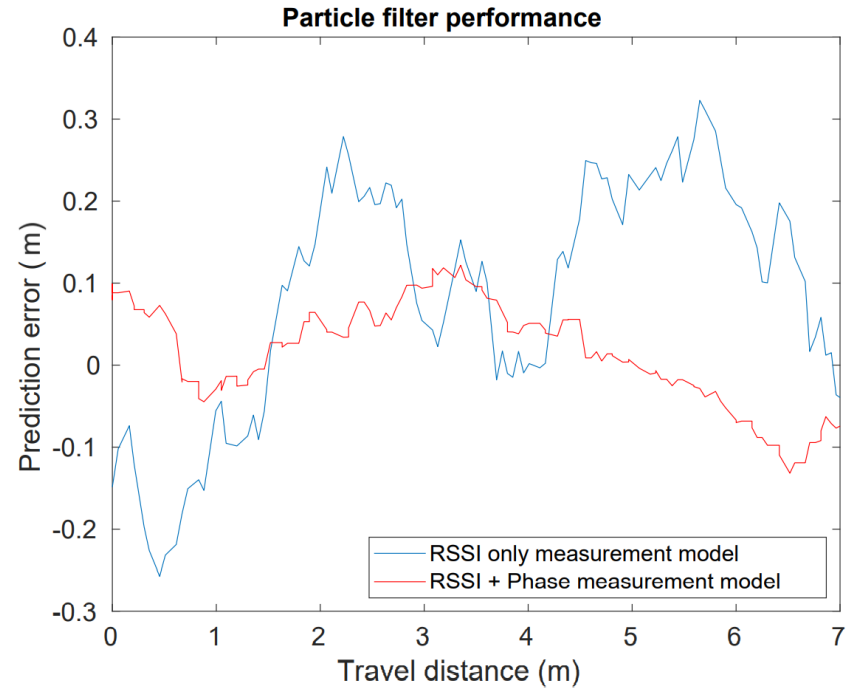

Fig. 10: Particle filter performance evaluation with different measurement models.

to evaluate different data collections and particle filter configurations. The best results were achieved when the robot travels at a maximum speed of 0.1 meters per second or less where RFID reader detects tags at a maximum rate of 50 tag readings per second. We have evaluated the particle filter performance with both RSSI data alone measurement model and with RSSI and Phase data combined data model. Fig. 10, 11 shows the performance evaluation of the particle filter for localization prediction values. The result comparisons show a significant improvement in the localization results when both RSSI and Phase data used in the particle filter measurement model. The proposed system has achieved a precision of 15 centimeter accuracy in localization results. Finally particle filter performance was evaluated with different number of particle configurations as shown in Fig. 12. The legend indicates the number of particles for each performance data curve. The results show that the maximum accuracy has been captured at 300 particles. Increasing it further (Fig. 12 $\mathrm{p}=400$ ) reduces the performance of the system and the error rate increases due to the prediction algorithms takes too long to process and respond back.

\section{COnClusion And Future Work}

This paper presented the developments of a robotic system for localizing inside the pipeline based on UHF-RFID signals. The robotic localization uses particle filter combined with Gaussian process algorithm. By integrating both RSSI and phase shift values into the particle filter model, the localization results are further enhanced. This scheme can be used for localization without the aid of odometry after creating the initial measurement model. The system was validated in lab experiments by using a pipe sample extracted from water pipe network. We have identified the best locations to deploy the RFID tags inside the pipe and the results conclude that the proposed system has a precision of 15 centimeter accuracy. In future, we intend to explore two RFID antenna readers for improving the accuracy and test the performance under different environmental conditions inside the pipeline. 


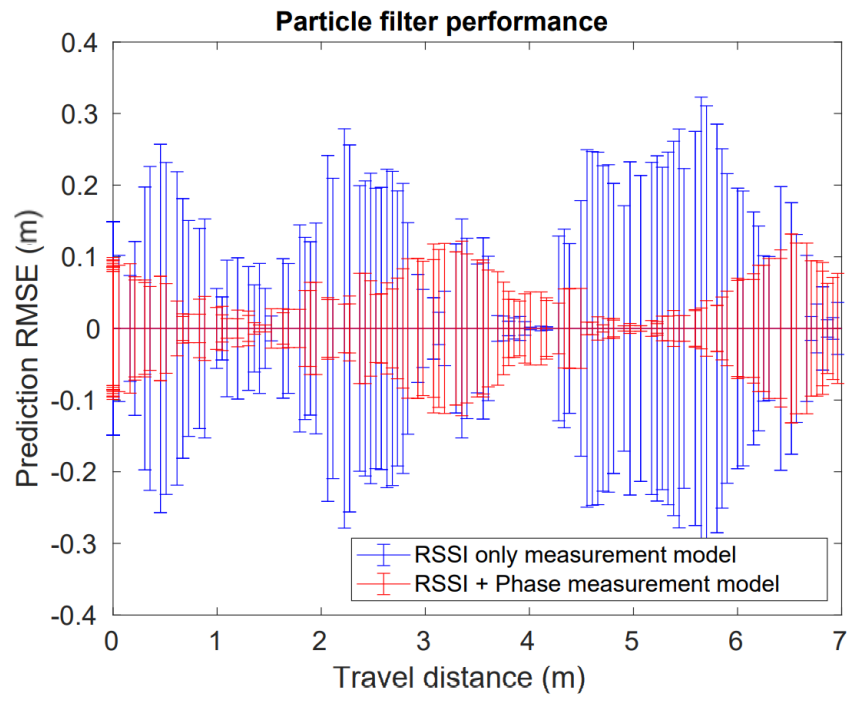

Fig. 11: Error boundary representation of the particle filter performance evaluation with different measurement models.

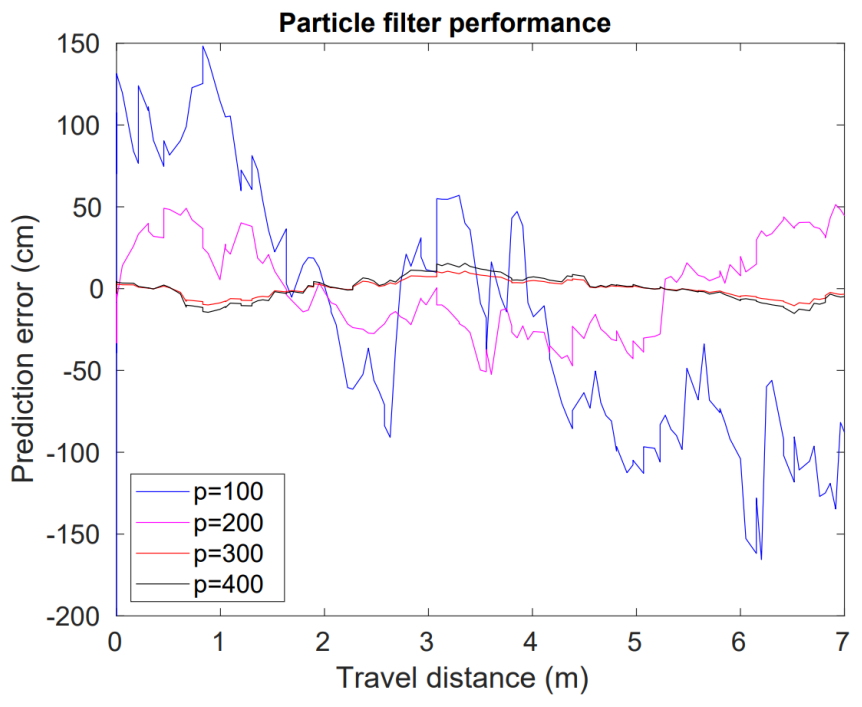

Fig. 12: Particle filter performance evaluation against the number of particles.

\section{ACKNOWLEDGMENT}

This work was supported by the UTS FEIT Tech Lab Blue Sky Research Grant. Special thanks to Sydney Water funded project, "Development of sensor suites and robotic deployment strategies for condition assessment of concrete sewer walls" for making the robot available for this work.

\section{REFERENCES}

[1] K. Thiyagarajan, S. Kodagoda, L. V. Nguyen, and R. Ranasinghe, "Sensor Failure Detection and Faulty Data Accommodation Approach for Instrumented Wastewater Infrastructures," IEEE Access, vol. 6, pp. $56562-56574,2018$.

[2] N. Ulapane, K. Thiyagarajan, J. Miro, and S. Kodagoda, "Surface representation of pulsed eddy current sensor signals for improved ferromagnetic material thickness quantification," IEEE Sensors Journal, vol. 21, no. 4, pp. 5413-5422, 2021.

[3] A. Gunatilake, L. Piyathilaka, S. Kodagoda, S. Barclay, and D. Vitanage, "Real-Time 3D Profiling with RGB-D Mapping in Pipelines Using Stereo Camera Vision and Structured IR Laser Ring," in 2019 14th IEEE Conference on Industrial Electronics and Applications (ICIEA), 2019, pp. 916-921.

[4] K. Thiyagarajan, S. Kodagoda, R. Ranasinghe, G. Iori, and D. Vitanage, "Robust Sensor Suite Combined with Predictive Analytics Enabled Anomaly Detection Model for Smart Monitoring of Concrete Sewer Pipe Surface Moisture Conditions," IEEE Sensors Journal, vol. 20, no. 15, pp. 8232-8243, 2020.

[5] M. Ams, P. Ha, S. Taheri, S. Clark, M. J. Withford, H. Bustamante, J. Gonzalez, and L. Vorreiter, "Fibre optic temperature and humidity sensors for harsh wastewater environments," in 2017 Eleventh International Conference on Sensing Technology (ICST), 2017, pp. 1-3.

[6] N. Giovanangeli, L. Piyathilaka, S. Kodagoda, K. Thiyagarajan, S. Barclay, and D. Vitanage, "Design and development of drillresistance sensor technology for accurately measuring microbiologically corroded concrete depths," in Proceedings of the 36th International Symposium on Automation and Robotics in Construction. IAARC, 2019, pp. 735-742.

[7] R. V. Canales and C. M. Furukawa, "Signal processing for corrosion assessment in pipelines with ultrasound pig using matched filter," in 2010 9th IEEE/IAS International Conference on Industry Applications - INDUSCON 2010, 2010, pp. 1-6.

[8] K. Thiyagarajan, S. Kodagoda, R. Ranasinghe, D. Vitanage, and G. Iori, "Robust sensing suite for measuring temporal dynamics of surface temperature in sewers," Scientific Reports, vol. 8, no. 1, p. $16020,2018$.

[9] D. Ellison, F. Sever, P. Oram, W. Lovins, P. Oram, W. Lovins, A. Romer, S. J. Duranceau, and G. Bell, "Global review of spray-on structural lining technologies," Water Research Foundation, Denver, Tech. Rep., 2010.

[10] A. Gunatilake, L. Piyathilaka, A. Tran, V. K. Vishwanathan, K. Thiyagarajan, and S. Kodagoda, "Stereo Vision Combined With Laser Profiling for Mapping of Pipeline Internal Defects," IEEE Sensors Journal, vol. 21, no. 10, pp. 11926-11934, 2021.

[11] S. R. Rusu, M. J. D. Hayes, and J. A. Marshall, "Localization in largescale underground environments with rfid," in 2011 24th Canadian Conference on Electrical and Computer Engineering(CCECE), 2011, pp. 001 140-001 143

[12] C. Li, L. Mo, and D. Zhang, "Review on uhf rfid localization methods," IEEE Journal of Radio Frequency Identification, vol. 3, no. 4, pp. 205$215,2019$.

[13] J. Virtanen, L. Ukkonen, T. Björninen, L. Sydänheimo, and A. Z. Elsherbeni, "Temperature sensor tag for passive uhf rfid systems," in 2011 IEEE Sensors Applications Symposium, 2011, pp. 312-317.

[14] E. M. Amin, M. S. Bhuiyan, N. C. Karmakar, and B. Winther-Jensen, "Development of a low cost printable chipless rfid humidity sensor," IEEE Sensors Journal, vol. 14, no. 1, pp. 140-149, 2014.

[15] I. Gammoudi, B. Aissa, M. Nedil, and M. M. Abdallah, "Cntrfid passive tag antenna for gas sensing in underground mine," in 2015 IEEE International Symposium on Antennas and Propagation USNC/URSI National Radio Science Meeting, 2015, pp. 1758-1759.

[16] A. Buffi, P. Nepa, and R. Cioni, "Sarfid on drone: Drone-based uhfrfid tag localization," in 2017 IEEE International Conference on RFID Technology Application (RFID-TA), 2017, pp. 40-44.

[17] F. Martinelli, "A robot localization system combining rssi and phase shift in uhf-rfid signals," IEEE Transactions on Control Systems Technology, vol. 23, no. 5, pp. 1782-1796, 2015.

[18] J. Zhang, Y. Lyu, J. Patton, S. C. G. Periaswamy, and T. Roppel, "Bfvp: A probabilistic uhf rfid tag localization algorithm using bayesian filter and a variable power rfid model," IEEE Transactions on Industrial Electronics, vol. 65, no. 10, pp. 8250-8259, 2018.

[19] Y. Xu, K. Xu, J. Wan, Z. Xiong, and Y. Li, "Research on particle filter tracking method based on kalman filter," in 2018 2nd IEEE Advanced Information Management,Communicates,Electronic and Automation Control Conference (IMCEC), 2018, pp. 1564-1568.

[20] N. Y. Ko and T. G. Kim, "Comparison of kalman filter and particle filter used for localization of an underwater vehicle," in 2012 9th International Conference on Ubiquitous Robots and Ambient Intelligence (URAI), 2012, pp. 350-352.

[21] C. E. Rasmussen, "Gaussian processes for machine learning," 2006.

[22] K. Thiyagarajan, P. Acharya, L. Piyathilaka, and S. Kodagoda, "Numerical modeling of the effects of electrode spacing and multilayered concrete resistivity on the apparent resistivity measured using wenner method," in 2020 15th IEEE Conference on Industrial Electronics and Applications (ICIEA), 2020, pp. 200-206. 